\title{
Optimal and optimal-linear control over lossy, distributed networks
}

\author{
Markus Kögel ${ }^{*, 1}$ Rainer Blind ${ }^{* *}$ Frank Allgöwer ${ }^{* *}$ \\ Rolf Findeisen* \\ * Institute for Automation Engineering, Otto-von-Guericke-University \\ Magdeburg, Germany.e-mail: \{markus.koegel, rolf.findeisen\}@ovgu.de. \\ ** Institute for Systems Theory and Automatic Control, University of \\ Stuttgart, Germany. e-mail: \{blind, allgower\}@ist.uni-stuttgart.de.
}

\begin{abstract}
We consider an optimal control problem for networked control systems, where the loop is closed via a lossy, distributed network with an acknowledgment mechanism. The network is distributed in the sense that there are different sets of sensors and actuators that each communicate individually with the controller. We assume that all packets, i.e., the measurement packets, the control packets and the acknowledgment packets are sent over the lossy network and thus are subject to loss. We derive suboptimal controllers with respect to a quadratic cost criterion for the general case and optimal controllers for the case that all states are perfectly measured over a single link. Additionally, we present stability criteria for both cases.
\end{abstract}

Keywords: Distributed Network, Linear Systems, Networked Control Systems, Optimal Control.

\section{INTRODUCTION}

During the last decades, packet based networks became very powerful and inexpensive to deploy and are increasingly used in control and estimation. Their possibly unreliable communication, e.g., packet loss or delay, needs to be considered in the controller design. There is an increasing trend to analyze and design Networked Control Systems (NCS) - systems, where the control loop is closed via a packet based network, see, e.g., Yang (2006); Hespanha et al. (2007).

In this work, an optimal control approach for NCS with lossy links using a quadratic cost criterion is presented. Assuming that acknowledgments are perfect, i.e., never lost, Sinopoli et al. (2005); Nilsson (1998); Garone et al. (2007) analyze such an optimal control problem, the latter one also for distributed networks. A similar approach for non-distributed networks without acknowledgments is considered in Imer et al. (2006) and Schenato et al. (2007). In Garone et al. (2008) optimal control over a distributed network with imperfect acknowledgments is considered. In a previous work, Kögel et al. (2010), we analyzed for a non-distributed network with imperfect acknowledgments robustness issues of such an optimal controller. All these works assume that all states are perfectly measurable, i.e., without measurement noise or that acknowledgments are never lost. In contrast, in Sinopoli et al. (2006) suboptimal control strategies for non-distributed networks without acknowledgments, but noisy measurements is studied. This suboptimal controller is called optimal-linear control, because it yields, under the given limitations, the best possible performance using a linear controller.

\footnotetext{
1 The researcher was supported in part by the International Max
} Plank Research School, Magdeburg, Germany.
These previous cited works are extended in this paper in two directions: considering a lossy acknowledgment mechanism as well as a distributed network. The main results of this work are 1) the derivation of strictly proper controllers, which are optimal, if we assume that all states are perfectly measured over a single link 2) optimal-linear controllers for the case of partial state measurements with additive noise and multiple output links.

The remainder of this paper is structured as follows. In Section 2, we formulate the problem and present the network model and networked plant. Afterwards, we discuss Kalman filtering in Section 3 and show that there is no separation principle due to the lossy acknowledgments. Next, we present the main results of this work: the design of strictly proper optimal (Section 4) and optimal-linear controllers (Section 5). In Section 6, some analytical stability criterions are derived. The results are illustrated by an example in Section 7 and summarized in Section 8.

Notation: Vectors are stated in bold face, $\times$ denotes the standard matrix product and $\otimes$ denotes the Hadamard or entry-wise matrix product. The identity matrix is $I$ and $1^{a \times b}$ is the matrix in $\mathbb{R}^{a \times b}$ with 1 in every entry. The Moore-Penrose pseudo-inverse of $M$ is denoted by $M^{\dagger}$.

\section{PROBLEM DESCRIPTION}

\subsection{Plant and network model}

We consider linear, time-invariant, discrete-time systems

$$
\begin{aligned}
\mathbf{x}_{k+1} & =A \mathbf{x}_{k}+B \mathbf{u}_{k}^{a}+\mathbf{w}_{k} \\
\mathbf{y}_{k}^{s} & =C \mathbf{x}_{k}+\mathbf{v}_{k},
\end{aligned}
$$

where $\mathbf{x}_{k} \in \mathbb{R}^{n}$ denotes the state of the plant, $\mathbf{u}_{k}^{a} \in \mathbb{R}^{p}$ the input applied by the actuators, $\mathbf{w}_{k} \in \mathbb{R}^{n}$ represents process noise, $\mathbf{y}_{k}^{s} \in \mathbb{R}^{q}$ denotes the output measured by the 


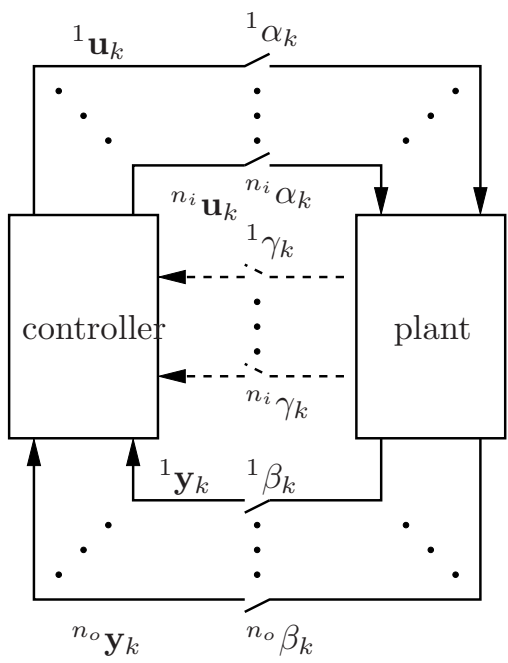

Fig. 1. Multi link Networked Control System.

sensors, and $\mathbf{v}_{k} \in \mathbb{R}^{q}$ is measurement noise. The system matrices $A, B$ and $C$ have the appropriate dimensions. It is assumed that $\mathbf{w}_{k}$ and $\mathbf{v}_{k}$ are mutually independent, zero mean Gaussian white noise with covariances $W$ and $V$. Furthermore, the initial condition $\mathbf{x}_{0}$ is Gaussian distributed with mean $\overline{\mathbf{x}}_{0}$ and covariance $P_{0}$ and is independent of the noise.

As illustrated in Fig. 1, the plant and controller are connected by an unreliable, distributed communication network. We assume that the sensors send the measurements $\mathbf{y}_{k}^{s}$ over the network to the controller. With respect to the actuators we assume that there is a two-way communication between the controller and the actuators: the controller sends the input $\mathbf{u}_{k}$ to the actuators and the actuators can inform the controller whether they received this input data or not.

It is assumed that the controller can individually communicate with different groups of actuators or sensors, respectively. Note that if there is only one link to all actuators or sensors, then the information sent over this link is either successfully transmitted or lost. In contrast, if there are multiple links related to specific sensors and actuators, then also partial information loss is possible.

In particular, there are $n_{i}$ so called input-data links and also $n_{i}$ input-acknowledgment links connecting the controller with the actuators. Furthermore, $n_{o}$ output links connect the sensors to the controller.

We model the loss behavior of each link using Bernoulli random processes, which are i.i.d. in time. In detail, the $j$ th input-data link is characterized by the process $\left\{{ }^{j} \alpha_{k}\right\}$. Moreover, $\left\{{ }^{m} \beta_{k}\right\}$ is the process modeling the $m$ th output link. Also the acknowledgment links $\left\{{ }^{j} \gamma_{k}\right\}$ are modeled using Bernoulli processes. Their definition will be given below in combination with the acknowledgment mechanism.

We define the probabilities of a successful transmission as $P\left({ }^{j} \alpha_{k}=1\right)={ }^{j} \bar{\alpha}$ and $P\left({ }^{m} \beta_{k}=1\right)={ }^{m} \bar{\beta}$. In addition, we assume that Bernoulli processes associated to different links types are mutually independent. Links of the same type can depend on each other, which is called non- independent links. In contrast, if $\left\{{ }^{j} \alpha_{k}\right\}$ is independent of $\left\{{ }^{l} \alpha_{k}\right\}$ and $\left\{{ }^{j} \gamma_{k}\right\}$ of $\left\{{ }^{l} \gamma_{k}\right\}$ for any $j, l \in\left\{1, \ldots, n_{i}\right\}, j \neq l$, then we call these independent input links. Similarly, if the output links are mutually independent, then these are called independent output links. In contrast to Garone et al. $(2007,2008)$ in our approach, the input links as well as output links can depend on themselves.

With respect to the links and system matrices we make the following assumptions. ${ }^{j} C \in \mathbb{R}^{q_{j} \times n}$ denotes the output matrix and ${ }^{j} \mathbf{v}_{k} \in \mathbb{R}^{q_{j}}$ the measurement noise of the sensor or sensors connected to output link $j$ with $q=$ $\sum_{j=1}^{n_{o}} q_{j}$. Additionally, $C$ and $\mathbf{v}_{k}$ are given by $C=$ $\left({ }^{1} C^{T}{ }^{2} C^{T} \ldots{ }^{n_{o}} C^{T}\right)^{T}, \mathbf{v}_{k}=\left({ }^{1} \mathbf{v}_{k}^{T}{ }^{2} \mathbf{v}_{k}^{T} \ldots{ }^{n_{o}} \mathbf{v}_{k}^{T}\right)^{T}$ and ${ }^{j} \mathbf{y}_{k}^{t}={ }^{j} C \mathbf{x}_{k}+{ }^{j} \mathbf{v}_{k}$. The covariance $V$ of the measurement noise is given by

$$
V=\left(\begin{array}{ccc}
{ }^{1,1} V & \ldots & { }^{1, n_{o}} V \\
\vdots & \ddots & \vdots \\
n_{o}, 1 & \ldots & n_{o}, n_{o} V
\end{array}\right)
$$

with ${ }^{i, j} V \in \mathbb{R}^{q_{i} \times q_{j}}$ and with $V=V^{T} \geq 0$. Using the output link $j$ the controller has access to the measurements via:

$$
{ }^{j} \mathbf{y}_{k}= \begin{cases}{ }^{j} C \mathbf{x}_{k}+{ }^{j} \mathbf{v}_{k}, & \text { if }{ }^{j} \beta_{k}=1 \\ \text { no measurement, } & \text { if }{ }^{j} \beta_{k}=0 .\end{cases}
$$

The actuators connected to the input link $j$ feature the inputs ${ }^{j} \mathbf{u}_{k}^{a} \in \mathbb{R}^{p_{j}}$ and the input matrix ${ }^{j} B \in \mathbb{R}^{n \times p_{j}}$. We assume that $p=\sum_{j=1}^{n_{i}} p_{j}, B=\left({ }^{1} B{ }^{2} B \ldots{ }^{n_{i}} B\right)$ and $\mathbf{u}_{k}^{a}=\left(\left({ }^{1} \mathbf{u}_{k}^{a}\right)^{T}\left({ }^{2} \mathbf{u}_{k}^{a}\right)^{T} \ldots\left({ }^{n_{i}} \mathbf{u}_{k}^{a}\right)^{T}\right)^{T}$. Note that these assumptions are without loss of generality.

Moreover, we assume that all actuators apply the so-called zero-input strategy

$$
{ }^{j} \mathbf{u}_{k}^{a}= \begin{cases}{ }^{j} \mathbf{u}_{k}, & \text { if }{ }^{j} \alpha_{k}=1 \\ \mathbf{0}, & \text { if }{ }^{j} \alpha_{k}=0 .\end{cases}
$$

So, if the actuators do not receive the control packets, then they apply zero input. Note that this scheme is quite common and used in Garone et al. (2007, 2008); Imer et al. (2006); Schenato et al. (2007); Sinopoli et al. (2005, 2006); Kögel et al. (2010), but is in general conservative, see Schenato (2009). Another, possibility is to hold inputs as considered in Nilsson (1998) or to allow more general schemes as in Kögel and Findeisen (2011).

In summary, the overall dynamics of the networked plant is given by (1) and

$$
\mathbf{x}_{k+1}=A \mathbf{x}_{k}+\sum_{j=1}^{n_{i}}{ }^{j} \alpha_{k}{ }^{j} B^{j} \mathbf{u}_{k}+\mathbf{w}_{k} .
$$

\subsection{Acknowledgment mechanism}

In order to inform the controller about the fate of the control packet and thus the input to the plant, the actuator sends an acknowledgment to the controller. There are positive and negative acknowledgments, cf. Leon-Garcia and Widjaja (2004), A positive acknowledgment, called ACK, is used to report a received control packet. A negative acknowledgment, called NAK, informs the controller of an unsuccessful transmission. If acknowledgments cannot get lost, one type is sufficient. Unfortunately, acknowledgments can also get lost and there will be differences, 


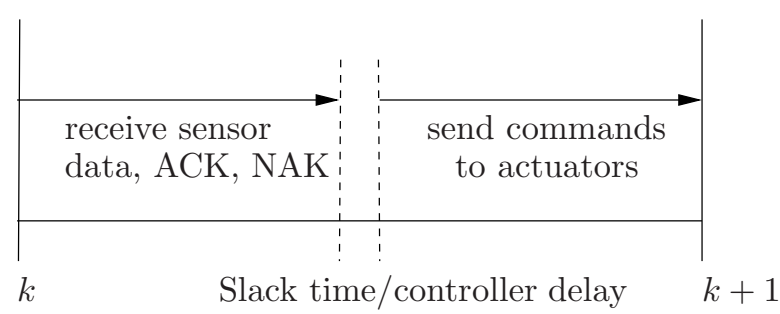

Fig. 2. Sampling time and possible network delay.

depending on the acknowledgments used. Note that it is impossible to communicate in real-time and reliable over a lossy channel, which is known as the two generals problems, see Leon-Garcia and Widjaja (2004). For example TCP is a reliable protocol, but not a real-time protocol.

We model the loss of each acknowledgment link $\left\{{ }^{j} \gamma_{k}\right\}$ using two Bernoulli processes: $\left\{{ }^{j} \theta_{k}^{A}\right\}$ for ACKs and $\left\{{ }^{j} \theta_{k}^{N}\right\}$ for NAKs, which are i.i.d. in time. So we have ${ }^{j} \gamma_{k}=$ ${ }^{j} \alpha_{k}{ }^{j} \theta_{k}^{A}+\left(1-{ }^{j} \alpha_{k}\right)^{j} \theta_{k}^{N}$. Moreover we assume that these processes are independent of the processes of the output and input-data link. As mentioned earlier processes of different acknowledgment links can depend on each other.

Using these setup we can consider different cases in one framework. Note that no acknowledgments are used if ${ }^{j} \bar{\theta}^{A}={ }^{j} \bar{\theta}^{N}=0, \forall j$, which is studied in Imer et al. (2006); Schenato et al. (2007); Sinopoli et al. (2006). Whereas ${ }^{j} \bar{\theta}^{A}=1$ or ${ }^{j} \bar{\theta}^{N}=1, \forall j$, i.e., perfect acknowledgments is assumed in Nilsson (1998); Garone et al. (2007); Imer et al. (2006); Schenato et al. (2007); Sinopoli et al. (2005). Garone et al. (2008); Kögel et al. (2010) analyze lossy acknowledgments, but both types of acknowledgments need to have the same loss rates, i.e., $j \bar{\theta}^{A}={ }^{j} \bar{\theta}^{N}, \forall j$ or, in the latter one, it is also possible that only one of the two acknowledgments is available.

The framework covers the case of different, nontrivial loss rates, which might result from, e.g., different timings.

\subsection{Controller specification}

We consider strictly proper controllers, i.e., controllers, which calculate $\mathbf{u}_{k}$ using only information available prior to $k$. In contrast, not strictly proper controllers use, in addition, the measurement at step $k$ to determine $\mathbf{u}_{k}$. Obviously, these controllers have more information available and thus might yield better performance. But the input at step $k$ obtained by the controller depends on the output at step $k$, which might be problematic, if the network or computational delay is not negligible. In Imer et al. (2006); Schenato et al. (2007); Sinopoli et al. (2005); Garone et al. (2007, 2008); Kögel et al. (2010) proper controllers have been considered and in Sinopoli et al. (2006); Kögel et al. (2010) strictly proper controllers.

In contrast, if we consider strictly proper controllers, then the network delay is not a critical issue as long as the sampling time is larger as the total network delay (round trip time) plus the computational delay, as simplified illustrated in Fig. (2). Moreover, as outlined in Kögel (2009) it is possible to retransmit packets as long as this takes place within the above mentioned timing bounds.
In the following, we denote by the sets $\mathcal{S}_{k}$ all information available prior to step $k+1$. These sets are defined by

$$
\begin{aligned}
\mathcal{S}_{-1} & =\left\{\overline{\mathbf{x}}_{0}, P_{0}\right\} \\
\mathcal{S}_{k} & =\left\{\mathcal{S}_{k-1}, \mathbf{y}_{k},\left({ }^{j} \gamma_{k},{ }^{j} \alpha_{k}{ }^{j} \gamma_{k}\right), j=1, \ldots, n_{i}\right\},
\end{aligned}
$$

where $\left({ }^{j} \gamma_{i},{ }^{j} \alpha_{i}{ }^{j} \gamma_{i}\right)$ is used to include the acknowledgment and its type (ACK/NAK). In consequence, a strictly proper controller uses the set $\mathcal{S}_{k}$ to compute $\mathbf{u}_{k+1}$ :

$$
\mathbf{u}_{k+1}=f\left(\mathcal{S}_{k}\right),
$$

i.e., only old information is used.

We focus on infinite horizon optimal control in this work. Specifically, we consider as performance the cost

$$
\begin{aligned}
& J_{\infty}=\limsup _{N \rightarrow \infty} \frac{1}{N} E\left\{\sum_{i=0}^{N-1}\left(\mathbf{x}_{i}^{T} Q \mathbf{x}_{i}+\left(\mathbf{u}_{i}^{a}\right)^{T} R \mathbf{u}_{i}^{a}\right)\right\} \\
& E\left\{\left(\mathbf{u}_{i}^{a}\right)^{T} R \mathbf{u}_{i}^{a}\right\}=E\left\{\sum_{j=1}^{n_{i}} \sum_{l=1}^{n_{i}}{ }^{j} \alpha_{k}{ }^{l} \alpha_{k}{ }^{j} \mathbf{u}_{k}^{T j}{ }^{j} l R^{l} \mathbf{u}_{k}\right\},
\end{aligned}
$$

where

$$
R=\left(\begin{array}{ccc}
{ }^{1,1} R & \ldots & { }^{1, n_{i}} R \\
\vdots & \ddots & \vdots \\
n_{i}, 1 & \ldots & { }^{n_{i}, n_{i}} R
\end{array}\right)
$$

and ${ }^{j, m} R \in \mathbb{R}^{p_{j} \times p_{m}}$, and $Q \in \mathbb{R}^{n \times n}, Q=Q^{T} \geq 0$, $R \in \mathbb{R}^{p \times p}, R=R^{T}>0$. The quadratic cost criterion weights only the applied inputs $\mathbf{u}_{k}^{a}$ - not the input $\mathbf{u}_{k}$ sent to the controller - and can be infinite.

\section{OPTIMAL ESTIMATION}

Note that there are two different sets of information available for estimation, which are represented by

$$
\begin{aligned}
\mathcal{S}_{-1}^{\star} & =\left\{\overline{\mathbf{x}}_{0}, P_{0}\right\}, \mathcal{U}_{k}^{\star}=\left\{\mathcal{S}_{k-1}^{\star}, \mathbf{y}_{k}\right\} \\
\mathcal{S}_{k}^{\star} & =\left\{\mathcal{U}_{k}^{\star}, \mathbf{u}_{k},\left({ }^{j} \gamma_{k},{ }^{j} \alpha_{k}{ }^{j} \gamma_{k}\right) ; j=1, \ldots, n_{i}\right\} .
\end{aligned}
$$

$\mathcal{S}_{k}^{\star}$ includes all information available prior to step $k+1$, in contrast, $\mathcal{U}_{k}^{\star}$ is the information available at step $k$. Moreover, the set $\mathcal{S}_{k}^{\star}$ includes the input $\mathbf{u}_{k}$ in contrast to the set $\mathcal{S}_{k}$. Consequently, there are two optimal estimates: the predicted estimate $\hat{\mathbf{x}}_{k \mid k-1}=E\left\{\mathbf{x}_{k} \mid \mathcal{S}_{k-1}^{\star}\right\}$ and the filtered estimate $\hat{\mathbf{x}}_{k \mid k}=E\left\{\mathbf{x}_{k} \mid \mathcal{U}_{k}^{\star}\right\}$. For this setup a detailed derivation of the Kalman filter can be found in Kögel (2009). The time update of the Kalman filter is

$$
\begin{aligned}
& \hat{\mathbf{x}}_{k+1 \mid k}=A \hat{\mathbf{x}}_{k \mid k}+\sum_{j=1}^{n_{i}}\left({ }^{j} \alpha_{k}{ }^{j} \gamma_{k}+\left(1-{ }^{j} \gamma_{k}\right)^{j} \epsilon_{k}\right)^{j} B^{j} \mathbf{u}_{k} \\
& \hat{\mathbf{e}}_{k+1 \mid k}=A \hat{\mathbf{e}}_{k \mid k}+\mathbf{w}_{k}+\sum_{j=1}^{n_{i}}\left(1-{ }^{j} \gamma_{k}\right)\left({ }^{j} \alpha_{k}-{ }^{j} \epsilon_{k}\right)^{j} B^{j} \mathbf{u}_{k} .
\end{aligned}
$$

where $\mathbf{e}_{k+1 \mid k}, \mathbf{e}_{k \mid k}$ denotes the error related to the estimates $\hat{\mathbf{x}}_{k+1 \mid k}, \hat{\mathbf{x}}_{k \mid k}$ and ${ }^{j} \epsilon_{k}=E\left\{{ }^{j} \alpha_{k} \mid{ }^{j} \gamma_{k}=0\right\}$. In the case of independent input links ${ }^{j} \epsilon_{k}$ is

$$
{ }^{j} \epsilon_{k}={ }^{j} \bar{\epsilon}=\frac{{ }^{j} \bar{\alpha}^{j} \bar{\theta}^{A}}{1-{ }^{j} \bar{\alpha}^{j} \bar{\theta}^{A}}+\frac{\left(1-{ }^{j} \bar{\alpha}\right)^{j} \bar{\theta}^{N}}{1-(1-j \bar{\alpha})^{j} \bar{\theta}^{N}} .
$$

We observe that the error $\mathbf{e}_{k+1 \mid k}$ depends on the input ${ }^{j} \mathbf{u}_{k}$, if there is no acknowledgment $\left({ }^{j} \gamma_{k}=0\right)$. So, the separation principle holds, if and only if, the controller always knows the behavior of the input links. 
Lemma 1. (Separation principle) The separation principle holds, if and only if, ${ }^{j} \bar{\alpha}=0,{ }^{j} \bar{\alpha}=1,{ }^{j} \bar{\theta}^{A}=1$ or ${ }^{j} \bar{\theta}^{N}=1$ for all $j$.

Due to the lack of the separation principle the input has an influence on the measurement update, which is not presented here for the general case.

If all states are perfectly measured over a single link, i.e., $n_{o}=1, C$ is invertible and $V=0$, then only the measurement update of the estimate is independent of the input. It can easily be obtained as

$$
\hat{\mathbf{x}}_{k \mid k}=\beta_{k} \mathbf{x}_{k}+\left(1-\beta_{k}\right) \hat{\mathbf{x}}_{k \mid k-1},
$$

using $\mathbf{x}_{k}=C^{-1} \mathbf{y}_{k}^{s}$. We can combine time and measurement update to get the optimal predictive estimator as

$$
\begin{aligned}
\hat{\mathbf{x}}_{k+1 \mid k}= & \left(1-\beta_{k}\right) A \hat{\mathbf{x}}_{k \mid k-1}+\beta_{k} A \mathbf{x}_{k} \\
& +\sum_{j=1}^{n_{i}}\left({ }^{j} \alpha_{k}{ }^{j} \gamma_{k}+{ }^{j}\left(1-\gamma_{k}\right)^{j} \epsilon_{k}\right)^{j} B^{j} \mathbf{u}_{k},
\end{aligned}
$$

with the initial estimate $\hat{\mathbf{x}}_{0 \mid-1}=\overline{\mathbf{x}}_{0}$.

\section{OPTIMAL CONTROL FOR A SPECIAL CASE}

First, we consider the case $n_{o}=1, C^{-1}$ exists and $V=0$. Theorem 2. (Optimal control for special case) Consider the system (1), (2), cost criterion (3) and assume that $n_{0}=1, C$ invertible and $V=0$. If the iteration

$$
\begin{aligned}
M_{k+1}= & (1-\bar{\beta})\left(A^{T} M_{k} A\right)+\left(A^{T} K_{k} B\right) \\
& \times[\alpha]\left([\alpha \alpha] \otimes\left(R+B^{T} K_{k} B\right)\right. \\
& \left.+[\alpha \phi] \otimes\left(B^{T} M_{k} B\right)\right)^{\dagger}[\alpha]\left(B^{T} K_{k} A\right) \\
K_{k+1}= & A^{T} K_{k} A+Q-M_{k+1}+(1-\bar{\beta}) \otimes\left(A^{T} M_{k} A\right) \\
M_{0}= & 0, K_{0}=I,
\end{aligned}
$$

converges, where

$$
\begin{aligned}
{[\alpha]=} & \operatorname{diag}\left({ }^{1} \bar{\alpha} \cdot I^{p_{1} \times p_{1}},{ }^{2} \bar{\alpha} \cdot I^{p_{2} \times p_{2}}, \ldots\right) \\
{[\alpha \alpha]=} & \left(\begin{array}{cc}
{ }^{1} \bar{\alpha} \cdot 1^{p_{1} \times p_{1}} & \ldots \\
E\left\{{ }^{1} \alpha_{k} \cdot{ }^{2} \alpha_{k}\right\} \cdot 1^{p_{2} \times p_{1}} & \ldots \\
\vdots & \ddots
\end{array}\right) \\
{[\alpha \phi]=} & \left(\begin{array}{cc}
E\left\{\left(1-{ }^{1} \gamma_{k-1}\right)^{2}\right. & \\
\left.\cdot\left({ }^{1} \alpha_{k-1}-{ }^{1} \epsilon_{k-1}\right)^{2}\right\} \cdot 1^{p_{1} \times p_{1}} & \ldots \\
E\left\{\left({ }^{1} \alpha_{k-1}-{ }^{1} \epsilon_{k-1}\right)\right. & \\
\cdot\left(1-{ }^{1} \gamma_{k-1}\right) \cdot\left(1-{ }^{2} \gamma_{k-1}\right) & \ldots \\
\left.\cdot\left({ }^{2} \alpha_{k-1}-{ }^{2} \epsilon_{k-1}\right)\right\} \cdot 1^{p_{2} \times p_{1}} & \\
\vdots & \ddots
\end{array}\right),
\end{aligned}
$$

then the optimal controller is given by

$$
\begin{aligned}
\mathbf{u}_{k}= & -G_{\infty} \hat{\mathbf{x}}_{k \mid k-1} \\
G_{\infty}=[\alpha]\left([\alpha \alpha] \otimes\left(R+B^{T} K_{\infty} B\right)\right. & \\
& \left.+[\alpha \phi] \otimes\left(B^{T} M_{\infty} B\right)\right)^{\dagger}[\alpha]\left(B^{T} K_{\infty} A\right),
\end{aligned}
$$

and the estimator (5). Moreover, the optimal cost is

$$
\begin{aligned}
J_{\infty} & =\operatorname{Tr}\left(\left(K_{\infty}+M_{\infty}\right) W\right) \\
M_{\infty} & =\lim _{k \rightarrow \infty} M_{k}, K_{\infty}=\lim _{k \rightarrow \infty} K_{k} .
\end{aligned}
$$

A detailed proof is avoided here, it can be found in detail in Kögel (2009).

Remark 3. (Implementation) Note that the optimal controller is a combination of a (stationary) Kalman filter and a linear state feedback, which can easily be implemented. In order to obtain the controller gain $G_{\infty}$ we need to numerically evaluate the iteration and check its convergence.

Next, we relate the finite, expected cost with upper bounds on the covariance in the next lemma.

Lemma 4. (Bounded covariance) If the cost per step (3) is finite and $\left(A, Q^{\frac{1}{2}}\right)$ observable, then there is an upper bound on $E\left\{\mathbf{x}_{k}^{T} \mathbf{x}_{k}\right\}$ and $E\left\{\hat{\mathbf{x}}_{k \mid k-1}^{T} \hat{\mathbf{x}}_{k \mid k-1}\right\}$.

In contrast, if the cost per step is infinite, then there is no upper bound on $E\left\{\mathbf{x}_{k}^{T} \mathbf{x}_{k}\right\}$.

This lemma is verified in Kögel (2009).

In contrast to Garone et al. (2008) we derived a strictly proper controller, while using a more general framework.

\section{OPTIMAL-LINEAR CONTROL}

In this section, we derive linear control laws for the general case. We aim to obtain a linear, strictly proper controller such that it has the best possible performance of all linear, strictly proper controllers, i.e., a so-called optimal-linear controller. Our networked control system fits into the framework of De Koning (1992).

Let us consider the system (2) and choose as plant input

$$
\mathbf{u}_{k}=-K \hat{\mathbf{x}}_{k}
$$

with the unknown controller gain $K$ and the estimate $\hat{\mathbf{x}}_{k}$. This estimate $\hat{\mathbf{x}}_{k}$ is obtained by the estimator

$$
\begin{aligned}
\hat{\mathbf{x}}_{k+1}= & A \hat{\mathbf{x}}_{k}+\sum_{j=1}^{n_{i}} E\left\{{ }^{j} \alpha_{k} \mid \mathcal{S}_{k}\right\}^{j} B^{j} \mathbf{u}_{k} \\
& +\sum_{j=1}^{n_{o}}{ }^{j} \beta_{k}{ }^{j} L\left({ }^{j} \mathbf{y}_{k}-{ }^{j} C \hat{\mathbf{x}}_{k}\right),
\end{aligned}
$$

with the yet unknown filter gains ${ }^{j} L, j=1, \ldots, n_{o}$. We want to choose ${ }^{j} L$ and $K$ such that the expected cost per step (3) is minimized. Note that considering a different estimator order, dynamic or input matrix does not increase the performance as mentioned in De Koning (1992).

Theorem 5. (Optimal-linear controller) Consider the system (1), (2) and the cost criterion (3). Let $Q>0, W>0$ or $\left(A, W^{\frac{1}{2}}\right)$ controllable, $\left(A, Q^{\frac{1}{2}}\right)$ observable and $V>0$, $R>0$. If the iteration

$$
\begin{aligned}
\bar{P}_{m+1} & =F_{1}\left(\bar{P}_{m}, \underline{P}_{m}, K_{m}, L_{m}\right), \bar{P}_{0}=I \\
\underline{P}_{m+1} & =F_{2}\left(\bar{P}_{m}, \underline{P}_{m}, K_{m}, L_{m}\right), \underline{P}_{0}=0 \\
\bar{\Lambda}_{m+1} & =F_{3}\left(\bar{\Lambda}_{m}, \underline{\Lambda}_{m}, K_{m}\right), \bar{\Lambda}_{0}=I \\
\underline{\Lambda}_{m+1} & =F_{4}\left(\bar{\Lambda}_{m}, \underline{\Lambda}_{m}, K_{m}, L_{m}\right), \underline{\Lambda}_{0}=0 \\
L_{m} & =F_{L}\left(\bar{P}_{m}\right), K_{m}=F_{K}\left(\bar{\Lambda}_{m}, \underline{\Lambda}_{m}\right),
\end{aligned}
$$

where

$$
\begin{aligned}
F_{1}= & A \bar{P}_{m} A^{T}-L_{m}[\beta] C \bar{P}_{m} A^{T}-A \bar{P}_{m} C^{T}[\beta] L_{m}^{T} \\
& +L_{m}\left([\beta \beta] \otimes\left(C \bar{P}_{m} C^{T}+V\right)\right) L_{m}^{T} \\
& +B\left([\alpha \phi] \otimes\left(K_{m} \underline{P}_{m} K_{m}^{T}\right)\right) B^{T}+W \\
F_{2}= & A \underline{P}_{m} A^{T}-B[\alpha] K_{m} \underline{P}_{m} A^{T}-A \underline{P}_{m} K_{m}^{T}[\alpha] B^{T} \\
& +L_{m}\left([\beta \beta] \otimes\left(C \bar{P}_{m} C^{T}+V\right)\right) L_{m}^{T} \\
& +B\left([\alpha \epsilon] \otimes\left(K_{m} \underline{P}_{m} K_{m}^{T}\right)\right) B^{T}
\end{aligned}
$$




$$
\begin{aligned}
F_{3}= & A^{T} \bar{\Lambda}_{m} A-A^{T} \bar{\Lambda}_{m} B[\alpha] K_{m}-K_{m}^{T}[\alpha] B^{T} \bar{\Lambda}_{m} A \\
& +K_{m}^{T}\left([\alpha \alpha] \otimes\left(B^{T} \bar{\Lambda}_{m} B+R\right)\right) K_{m} \\
& +K_{m}^{T}\left([\alpha \phi] \otimes\left(B^{T} \underline{\Lambda}_{m} B\right)\right) K_{m}+Q \\
F_{4}= & A^{T} \underline{\Lambda}_{m} A-C^{T}[\beta] L_{m}^{T} \underline{\Lambda}_{m} A-A^{T} \underline{\Lambda}_{m} L_{m}[\beta] C \\
& +K_{m}^{T}\left([\alpha \alpha] \otimes\left(B^{T} \bar{\Lambda}_{m} B+R\right)\right) K_{m} \\
& +K_{m}^{T}\left([\alpha \phi] \otimes\left(B^{T} \underline{\Lambda}_{m} B\right)\right) K_{m} \\
& +C^{T}\left([\beta \beta] \otimes\left(L_{m}^{T} \underline{\Lambda}_{m} L_{m}\right)\right) C \\
F_{K}= & \left([\alpha \alpha] \otimes\left(B^{T} \bar{\Lambda}_{m} B+R\right)\right. \\
& \left.+[\alpha \phi] \otimes\left(B^{T} \underline{\Lambda}_{m} B\right)\right)^{\dagger}[\alpha]\left(B^{T} \bar{\Lambda}_{m} A\right) \\
F_{L}= & A \bar{P}_{m} C^{T}[\beta]\left([\beta \beta] \otimes\left(C \bar{P}_{m} C^{T}+V\right)\right)^{\dagger},
\end{aligned}
$$

with $[\alpha],[\alpha \alpha],[\alpha \phi]$ as given in Theorem 2 and

$$
\begin{aligned}
& {[\alpha \epsilon]=\left(\begin{array}{cc}
E\left\{\left({ }^{1} \alpha_{k} \cdot{ }^{1} \gamma_{k}+\left(1-{ }^{1} \gamma_{k}\right) \cdot{ }^{1} \epsilon_{k}\right)^{2}\right\} & \ldots \\
\cdot 1^{p_{1} \times p_{1}} & \ldots \\
E\left\{\left({ }^{1} \alpha_{k} \cdot{ }^{1} \gamma_{k}+\left(1-{ }^{1} \gamma_{k}\right) \cdot{ }^{1} \epsilon_{k}\right)\right. & \\
\left.\cdot\left({ }^{2} \alpha_{k} \cdot{ }^{2} \gamma_{k}+\left(1-{ }^{2} \gamma_{k}\right) \cdot{ }^{2} \epsilon_{k}\right)\right\} 1^{p_{2} \times p_{1}} & \cdots \\
\vdots & \ddots
\end{array}\right)} \\
& {[\beta \beta]=\left(\begin{array}{cc}
{ }^{1} \bar{\beta} \cdot 1^{q_{1} \times q_{1}} & \cdots \\
E\left\{{ }^{1} \beta \cdot{ }^{2} \beta\right\} \cdot 1^{q_{2} \times q_{1}} & \cdots \\
\vdots & \ddots
\end{array}\right),}
\end{aligned}
$$

converges as $m \rightarrow \infty$, then the optimal-linear controller of the form $(7),(8)$ is uniquely given by the gains

$$
L=\lim _{m \rightarrow \infty} L_{m}=\left({ }^{1} L^{2} L \ldots\right), K=\lim _{m \rightarrow \infty} K_{m} .
$$

Moreover, the expected cost per step can be obtained by

$$
\begin{aligned}
J_{\infty} & =\operatorname{Tr}\left(\begin{array}{cc}
Q(\bar{P}+\underline{P}) & 0 \\
0 & K^{T}([\alpha \alpha] \otimes R) K \underline{P}
\end{array}\right) \\
\bar{P} & =\lim _{m \rightarrow \infty} \bar{P}_{m}, \underline{P}=\lim _{m \rightarrow \infty} \underline{P}_{m} .
\end{aligned}
$$

In addition, $E\left\{\left(\begin{array}{c}\mathbf{x}_{k} \\ \hat{\mathbf{x}}_{k}\end{array}\right)\left(\begin{array}{c}\mathbf{x}_{k} \\ \hat{\mathbf{x}}_{k}\end{array}\right)^{T}\right\}$ is bounded.

Finally the iteration diverges, if and only if, there is no stabilizing linear controller.

We refer to Kögel (2009) for the proof.

Remark 6. (Convergence of iteration) Checking the convergence of the iteration and thus stability in the mean square sense can be done similarly as in Sinopoli et al. (2006); De Koning (1992): numerically.

\section{STABILITY CRITERIONS}

In this section, we analyze the convergence of the iterations of Theorem 2 and 5, and thus the existence of mean square stabilizing controllers. First we consider the general case. Corollary 7. (Influence of covariance and weightings on existence of controllers)

If for one set $(Q, W, R, V)$ satisfying the conditions of Theorem 5 , there exists a stabilizing, optimal-linear controller (Theorem 5), then it exists for every such set.

If for $n_{o}=1, V=0, C$ invertible and a set $(Q, W, R)$ satisfying the conditions of Theorem 2 there exists a stabilizing, optimal controller (Theorem 2), then it exists for every such set $(Q, W, R)$.

Proof. This corollary follows directly from (De Koning, 1992, Theorem 3) and the fact that the controller obtained in Theorem 2 is linear.
Now let us consider the case of single links.

Theorem 8. (Existence of optimal and optimal-linear controller for single link systems) Assume $n_{i}=n_{o}=1, V=0$, $C$ invertible and let $\lambda_{\max }$ be the spectral radius of $A$. If $Q, R, W, V$ satisfy the assumptions of Theorem 5 , then a necessary condition for the convergence of the existence of a stabilizing optimal-linear controller is

$$
\lambda_{\max }^{2}\left(1-\frac{\bar{\alpha}}{1-(1-\bar{\beta}) \lambda_{\max }^{2}}>0\right.
$$

where $\phi=\left(1-\bar{\theta}^{A}\right) \cdot(1-\bar{\epsilon})^{2}+\frac{(1-\bar{\alpha}) \cdot\left(1-\bar{\theta}^{N}\right) \cdot \bar{\epsilon}^{2}}{\bar{\alpha}}$.

If $B$ is invertible, then the conditions are also sufficient. For $n_{o}=1, V=0, C$ invertible, $\left(A, Q^{\frac{1}{2}}\right)$ observable, $R>0, V=0$, and $W$ arbitrary, the above conditions hold also for the optimal controller presented in Theorem 2 .

The proof is based on the results of Garone et al. (2008); Schenato et al. (2007) and can be found in Kögel (2009).

\section{EXAMPLE}

We consider a linearized model of the seesaw inverted pendulum system given in Ramos et al. (1998), see Fig. 3. This unstable system uses two carts on a seesaw, to stabilize an inverted pendulum mounted onto one of this cart. The states are $\mathbf{x}=\left(d_{1}, \psi, d_{2}, \delta, \dot{d}_{1}, \dot{\psi}, \dot{d}_{2}, \dot{\delta}\right)$. Each cart is connected to a motor with a belt drive. The inputs are the voltages of the motors $\mathbf{u}=\left(U_{1}, U_{2}\right)$. Consequently, the continuous-time system is given by

$$
\begin{aligned}
\dot{\mathrm{x}} & =A \mathbf{x}+B \mathbf{u} \\
A & =\left(\begin{array}{cc}
0^{4 \times 4} & I^{4 \times 4} \\
A_{21} & A_{22}
\end{array}\right), B=\left(\begin{array}{c}
0^{4 \times 2} \\
B_{2}
\end{array}\right), \\
A_{21} & =\left(\begin{array}{cccc}
-0.976 & 8.99 & -1.43 & 0 \\
6.99 & 5.88 & 10.2 & 0 \\
-0.976 & 8.99 & -1.43 & -4.53 \\
-6.99 & -5.88 & -10.2 & 47.0
\end{array}\right), \\
A_{22} & =\left(\begin{array}{cccc}
16.4 & 0 & -0.224 & 0 \\
1.60 & 0 & 1.60 & 0 \\
-0.224 & 0 & -7.44 & 0 \\
-1.60 & 0 & 51.3 & 0
\end{array}\right), B_{2}=\left(\begin{array}{ccc}
3.74 & 0.051 \\
-0.37 & -0.37 \\
0.051 & 1.70 \\
0.367 & -11.7
\end{array}\right)
\end{aligned}
$$

We use a sampling time of $20 \mathrm{~ms}$ to discretize the system resulting in a spectral radius of 1.4 of $A$.

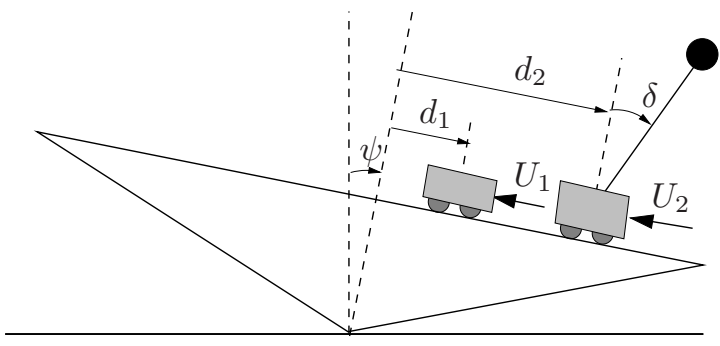

Fig. 3. Seesaw inverted pendulum system.

We use different setups. First, we consider that all states are measured, i.e., $C=I^{8 \times 8}$ and assume that there are a single input and single output links (case 1). Next, in case 2 , we use two input links and a single output link. Moreover we consider, that only positions and angles are measured, 
Table 1. Optimal/Optimal-linear control. Expected cost per step.

\begin{tabular}{c||ccc|ccc}
\multicolumn{1}{c||}{ Meas. noise } & \multicolumn{3}{c|}{$V=0$} & \multicolumn{3}{c}{$V=0.1 I$} \\
\hline Acknow. & Both & ACK & NAK & Both & ACK & NAK \\
\hline$I$ & 3.81 & 4.55 & 3.85 & 4.35 & 5.19 & 4.38 \\
$I I$ & 3.81 & 4.54 & 3.84 & 4.34 & 5.18 & 4.38 \\
III & 186 & 366 & 192 & 275 & 647 & 287 \\
$I V$ & 185 & 366 & 191 & 274 & 646 & 286
\end{tabular}
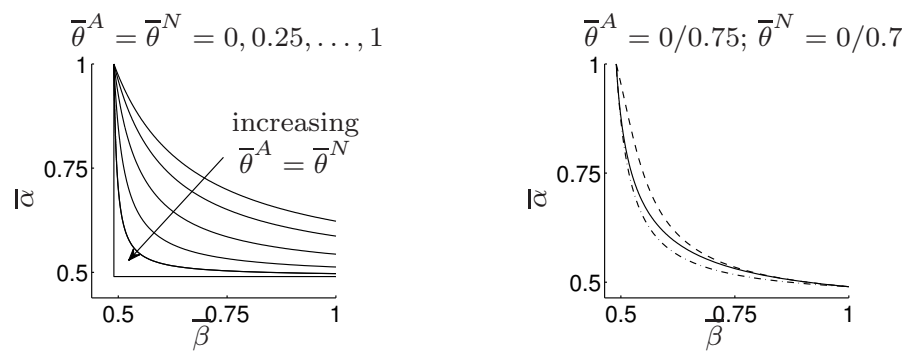

Fig. 4. Boundaries of Stability Areas.

i.e., $C=\left(I^{4 \times 4} 0^{4 \times 4}\right)$ and two input links and a single output link are used (case 3 ) or two input links and four output links are used: for every measurement a separate link (case 4). All links are assumed to be independent. For each of these cases we considered zero measurement noise $V=0$ and noisy measurement $V=0.1 I$ as well as availability of only ACKs, only NAKs or both of them. We use $W=0.1 I$ and $Q=R=10^{-3} I$ and a probability of a successful transmission of 0.85 for every link.

Note that in the first two cases we can obtain optimal controllers for the noise free case (Theorem 2). For all other cases the obtained controller are only guaranteed to be optimal-linear (Theorem 5).

Table 1 shows the performance for the different setups, which were verified using Monte Carlo simulations. We observe that measurement of only the partial state leads to a significant performance drop. In addition, noisy measurements decrease the performance. Moreover, we observe that if we use only one acknowledgment type, then using only NAKs yields in this case a better control performance.

Let us again consider the single link setup, case 1 . In this case we can use the results obtained in Theorem 8 to determine necessary stability criteria ( $B$ is not invertible). In Fig. 4 we illustrated the boundary of the necessary stability areas in the $\bar{\alpha}-\bar{\beta}$ plane. The areas described by the condition are above the boundaries and do not include them: for any point below the boundary there exists no stabilizing controller. In the left plot we assume $\bar{\theta}^{A}=\bar{\theta}^{N}=$ $0,0.25,0.5,0.75,0.9,1$. The right plot compares the three acknowledgment possibilities using $\bar{\theta}^{A}=0 / 0.75 ; \bar{\theta}^{N}=$ $0 / 0.75$, where the dashed line is used for only ACKs, the solid line for only NAKs and the dash dotted line for ACKs and NAKs.

\section{CONCLUSIONS}

Optimal and optimal-linear control of a plant connected to the controller by a distributed, lossy network is the topic of this work. Due to the network loss, there is no separation principle. We obtain optimal-linear controllers for the case of partial, noisy state measurement and optimal controllers for the case of perfect measurement of all states over a single link. In addition, we present analytical stability criterions for the case of single links.

\section{REFERENCES}

De Koning, W.L. (1992). Compensatability and Optimal Compensation of Systems with White Parameters. IEEE Transactions on Automatic Control, 37(5), 579588.

Garone, E., Sinopoli, B., and Casavola, A. (2008). LQG Control over Lossy TCP-like Networks with Probabilistic Packet Acknowledgements. In Proceedings of the $4^{7}$ th IEEE Conference on Decision and Control, 2686-2691. Garone, E., Sinopoli, B., Goldsmith, A., and Casavola, A. (2007). LQG Control for Distributed Systems over TCPlike Erasure Channels. In Proceedings of the 46th IEEE Conference on Decision and Control, 44-49.

Hespanha, J.P., Naghshtabrizi, P., and Xu, Y. (2007). A survey of recent results in networked control systems. Proc. of IEEE Special Issue on Technology of Networked Control Systems, 95(1), 138-162.

Imer, O.C., Yuksel, S., and Basar, T. (2006). Optimal control of LTI systems over unreliable communication links. Automatica, 42, 1429-1439.

Kögel, M.J. and Findeisen, R. (2011). Robust suboptimal control over lossy networks using extended input schemes. Submitted to IEEE MSC 2011.

Kögel, M.J. (2009). On optimal control over networks with lossy links. Diploma thesis, University of Stuttgart. URL http://elib.uni-stuttgart.de/ opus/volltexte/2010/5106/.

Kögel, M.J., Blind, R., and Allgöwer, F. (2010). Optimal Control over Unreliable Networks with Uncertain Loss Rates. In Proceedings of the 2010 American Control Conference, 3672-3677.

Leon-Garcia, A. and Widjaja, I. (2004). Communication Networks. McGraw Hill, Boston.

Nilsson, J. (1998). Real-Time Control Systems with Delays. Ph.D. thesis, Lund Institute of Technology.

Ramos, L., Castillo-Toledo, B., and Negretc, S. (1998). Nonlinear regulation of a seesaw inverted pendulum. In Proceedings of the 1998 IEEE International Conference on Control Applications, 1399 -1403.

Schenato, L. (2009). To Zero or Hold Inputs with Lossy Links. IEEE Transactions on Automatic Control, 54(5), 1093-99.

Schenato, L., Sinopoli, B., Franceschetti, M., Poolla, K., and Sastry, S.S. (2007). Foundations of Control and Estimation over Lossy Networks. Proceedings of the IEEE, 95(1), 163-187.

Sinopoli, B., Schenato, L., Franceschetti, M., Poolla, K., and Sastry, S.S. (2005). Optimal Control with Unreliable Communication: the TCP Case. In Proceedings of the 2005 American Control Conference, 3354-3359.

Sinopoli, B., Schenato, L., Franceschetti, M., Poolla, K., and Sastry, S.S. (2006). Optimal Linear LQG Control over Lossy Networks without Packet Acknowledgement. In Proceedings of the 45th IEEE Conference on Decision and Control, 392-397.

Yang, T. (2006). Networked control system: a brief survey. IEE Proceedings - Control Theory and Applications, 103, $403-412$. 